
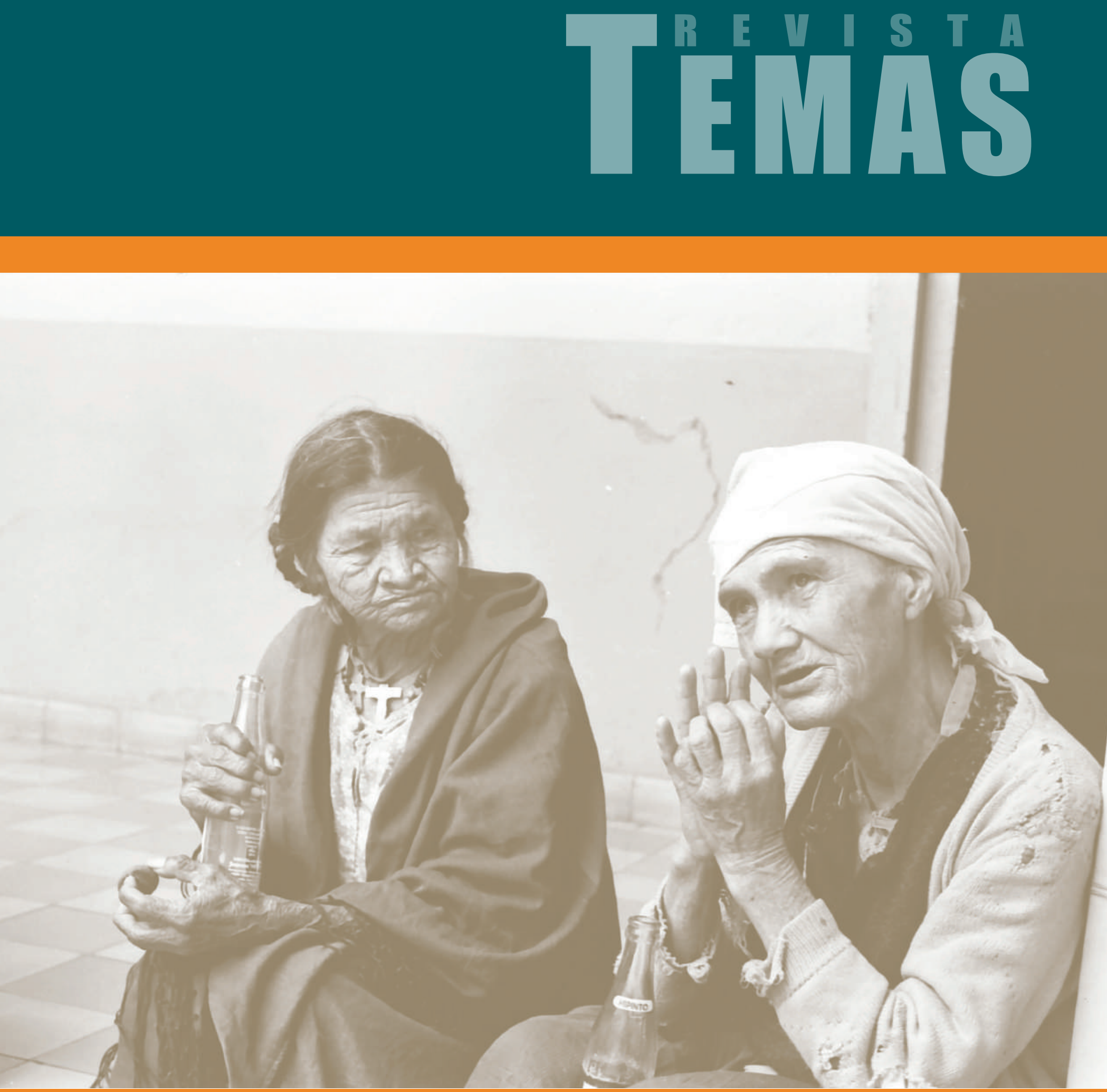



\section{DE LA TEORÍA DE COMPETENCIAS EN MATEMÁTICAS, A UNA PROPUESTA DE TRABAJO DE AULA CON SITUACIONES PROBIEMA1}

(Recepción: Mayo 31 de 2013- Aceptación: Agosto 01 de 2013)

Judith del Carmen Bertel Behaine*

\section{Resumen}

Con este estudio, se pone a consideración de los docentes de matemáticas de los distintos niveles, una propuesta de trabajo de aula orientada hacia el desarrollo de la competencia comunicativa en matemáticas a través de situaciones problema; ésta se fundamenta en una nueva conceptualización y caracterización de lo que asume como situación problema, enfatiza y privilegia el uso de los conocimientos en situaciones de contexto. El estudio se desarrolla en el grado 6으 de la institución educativa La Unión en el marco de la investigación acción, se abordó una situación problema como pretexto, los resultados permitieron evidenciar, que es posible que los estudiantes desarrollen competencias comunicativas en matemáticas a través del abordaje de situaciones problema que provienen de su cotidianidad.

\section{Palabras claves}

Problemas, Competencias, Matemáticas, Estudiantes

\section{ABOUT THE THEORY OF SKILIS IN MATHEMATIES, THE PROPOSAL FOR A CLASSROOM WORK WITH PROBLEM SITUATIONS}

\section{Alustract}

With this study, we offer mathematics teachers at different levels, a proposal for classroom work oriented towards the development of a communicative competence in mathematics through problem situations. It is based on a new conceptualization and characterization of the assumed problem situation and stresses and favors the use of knowledge in context situations. The study takes place in the 6th grade of the school La Union in the framework of action research, addressing a problem situation as a pretext. The results demonstrate that it is possible for the students to develop communication skills in mathematics by addressing problem situations that come from everyday life.

\section{Keywords}

Problems, Skills, Math, Students

1 Investigación institucional con apoyo del grupo de investigación (PROPED) realizada en el año del 2012 como experiencia de aula. Magíster en Educación. judithbertel @gmail.com 


\section{Introducción}

En las últimas décadas, las políticas educativas han estado dirigidas al mejoramiento cualitativo de la educación, con la pretensión de llegar a formar ciudadanos competentes, que ante la realidad problemática del país, realicen acciones y asuman una actitud reflexiva y comprometida con su desarrollo, de tal manera que sean capaces de tomar decisiones y proponer alternativas de solución ante las dificultades que se les presenten, haciéndolos verdaderos miembros activos de una comunidad, con un sincero sentido de pertenencia hacia ella.

Para lograr alcanzar las metas trazadas en lo pedagógico por el del Ministerio de Educación Nacional, se necesita que el trabajo realizado en el aula se reoriente y adquiera un nuevo matiz, en el sentido de cambiar las clases magistrales - donde muchas veces es sólo el docente quien tiene la última palabra y los alumnos poco tienen que decir y aportar con respecto de su propio aprendizaje - por procesos de formación que brinden espacios donde los alumnos tengan la oportunidad de debatir, dialogar, preguntar, argumentar y proponer. Que ante situaciones problema que se les presenten, tengan el espacio y la oportunidad para sentar su posición y tomar decisiones con respecto a ella, en pocas palabras, comprometerse con su propio aprendizaje (Bonilla, 1999).

Específicamente en el área de matemáticas, se les exige que pongan en práctica la competencia comunicativa, es decir, frente a una determinada situación problema, el estudiante debe desarrollar acciones interpretativas, argumentativas y propositivas que lo conlleven a encontrarle la mejor solución.

Con el ánimo de apoyar y contribuir con este nuevo trabajo de formación auspiciado por el MEN y de evaluación propuesto por el ICFES, se llevó a cabo esta investigación, que tuvo como objetivo principal, proponer una experiencia de aula orientada al desarrollo de la competencia comunicativa en el área de matemáticas a través de una situación problema. Específicamente se trabajó en desarrollar la competencia comunicativa con sus manifestaciones interpretativa, argumentativa y propositiva en el eje conceptual de aleatoriedad. La investigación se desarrolló en la Institución educativa La Unión de la ciudad de Sincelejo en el grado 60, para esto se contó con la colaboración de los estudiantes del curso y de algunos docentes y administrativos de la institución.

Con este trabajo los docentes y en general todos las personas que colaboraron en él, quisieron aportar desde lo pedagógico, una propuesta de trabajo de aula que responda a las pretensiones del MEN y el ICFES y que, a la vez, posibilite una nueva cultura de trabajo más acorde con las actuales exigencias del país.

\section{Marco Teórico}

Los lineamientos en el área de matemáticas presentan una propuesta, la cual se caracteriza por dar pautas que sirven a las instituciones educativas para que diseñen, elaboren, ejecuten y desarrollen el currículo. Para tener éxito en esta nueva visión curricular, se plantea en la propuesta de los lineamientos curriculares, que las matemáticas sean vistas como construcción humana, donde predomine la visión global de los estudiantes sobre el saber matemático y su utilización consecuente en su vida diaria. Para abordar el estudio de las matemáticas y más específicamente de la matemática escolar, es necesario tener en cuenta, el tipo de ciudadano que se piensa formar, la relación entre competencia y matemática escolar y por último el hacer 
matemático como actividad social, todo esto abordado desde la perspectiva de los lineamientos curriculares ( MEN, 1998).

Para que sea posible la matematización (Calderón, 2001) a partir de la realidad del estudiante y que verdaderamente le sirva en su vida cotidiana, es necesario que la escuela reestructure los currículos en matemática, para fortalecer con ello el desarrollo de competencias, que desde la perspectiva del ICFES (1999) significa un "saber hacer en contexto", la cual se evidencia a través de la resolución de problemas, ya que abordar un problema requiere ubicar el contexto y establecer de qué se dispone para encontrar una solución posible. En este proceso de significación, no se desconoce el hecho, que generalmente las situaciones problema y sus posibles soluciones son socialmente compartidas, es decir, están validadas en el contexto de la matemática escolar. Lo que implica necesariamente la exposición de los estudiantes ante situaciones reales de su vida cotidiana para que, a partir de ahí empiecen a buscar los posibles caminos, que le posibiliten resolver los interrogantes que serán superados, a partir de sus saberes previos y la interiorización de saberes elaborados. Visto desde esta perspectiva, los estudiantes se convierten en productores y no repetidores de conocimiento, lo cual propicia la investigación formativa, que se da sólo cuando en la práctica se vivencian los postulados teóricos, se reconceptualizan y se crean nuevas dimensiones del conocimiento (Bonilla, 1999).

Como la matemática es producto de una construcción humana y, por lo tanto, no marginable de la cotidianidad, (Beltrán, 1998) se convierte en un recurso para el aprendizaje en el uso, el cual consiste en poner en juego un saber frente a un problema contextualizado en la realidad del estudiante, de tal forma que en el transcurso de la resolución de este problema se genere un nuevo saber en el estudiante. Esto hace aparecer en el ámbito de la matemática escolar el concepto de competencia. En esa misma idea el ICFES (1999) plantea las competencias comunicativas en matemáticas. Tales competencias se manifiestan en tres acciones: interpretativa, argumentativa y propositiva, y, el mismo ICFES (1999) aclara que tales acciones no tienen jerarquía, ya que en el momento de dar cuenta por una de ellas, las otras dos aunque no se hagan visibles, también se despliegan internamente en el individuo.

\section{Marco conceptual}

Los problemas contextualizados, fundamentados desde los principios de la resolución de problemas, son, según el MEN (1998) la actividad más próxima al pensamiento cotidiano $y$, por ende, el medio privilegiado para la valoración y exploración significativa de los logros en la competencia matemática de los estudiantes, es en los problemas contextualizados donde los alumnos se encuentran con la posibilidad de significar desde las matemáticas el conocimiento que han logrado aprehender y construir a través de sus acciones interpretativas, argumentativas y propositivas, además, porque a través de este tipo de problemas, la construcción y el uso del conocimiento surge como una actividad natural en el trabajo de aula.

Conforme al ICFES (1999), la competencia comunicativa se evidencia a partir de enfrentar situaciones problema, porque en ellas se indaga por los usos e interpretaciones que de los objetos matemáticos haga el estudiante en un momento dado. Estas situaciones, que a partir de una primera mirada se pueden comparar con los problemas contextualizados, posibilitan la enseñanza, el aprendizaje y la evaluación de conceptos matemáticos, así como la puesta en práctica de procedimientos y estrategias para la resolución de problemas. 
El querer llevar al aula actividades que propicien de manera natural las acciones de interpretar, argumentar y proponer en matemáticas mediante la resolución de problemas, trae consigo construir una nueva u otra forma de trabajo de aula, pero este nuevo trabajo debe ser el resultado de una nueva labor, guiada por las orientaciones plasmadas en la nueva visión que se tiene de la matemática en la escuela (Santos, 1997) y en los lineamientos curriculares; estas orientaciones en el contexto de la matemática escolar se convierten en principios fundamentales para la reorganización del currículo de matemáticas, de tal manera que esté acorde con el nuevo contexto educativo en Colombia.

En relación con las consideraciones existentes en los lineamientos curriculares en torno a la nueva estructura curricular para el área de matemáticas, las situaciones problema son propuestas como un contexto amplio para desarrollar la actividad matemática en el aula. Además, ellas desde la visión del ICFES (1999), permiten vincular tanto los ejes conceptuales como las acciones que se quieren privilegiar en el uso del conocimiento matemático, pero es necesario pensar las situaciones problema de una manera más amplia que la existente y en la cual esté presente el énfasis en el uso del conocimiento. Así, conceptualizar la nueva situación problema desde la pretensión de este trabajo, es un paso obligado, porque el tener una idea clara de lo que se entiende por situación problema orientada al desarrollo de competencia, permite visionar hacia el futuro lo que hay que hacer para hacer de las clases espacios donde se desencadenen los procesos de aprendizajes esperados (Hernández, 1998).

Consecuentemente con lo anterior, se entiende una situación problema como:
La dinámica que se vivencia al interior de un aula de clases a partir de una propuesta de trabajo presentada por el profesor a unos estudiantes mediante un texto, en el que aparece un contexto hipotético o no y respecto del cual se formulan preguntas o tareas de carácter no matemático que comprometen la afectividad e interés del alumno y, además, al ser abordadas, posibilitan, con base en los conocimientos ya presentes en los estudiantes, procesos de búsqueda de explicación a los interrogantes planteados, que devienen construcción y constitución de nuevo conocimiento matemático, en y desde los estudiantes, en términos de conceptos, representaciones y procedimientos matemáticos, que potencian esa actitud hacia la producción de conocimientos y aprendizaje en el uso, de las matemáticas.

\section{Caracterización de situación problema}

La idea de situación problema que se propone, desde las consideraciones teóricas anteriormente propuestas, contiene unas relaciones que se estructuran a partir de: un contexto, unas formas de la actividad y de la acción en el aula, un proceso de búsqueda de explicación, momentos de evaluación y momentos para la institucionalización del saber. Caracterizando a cada uno de ellos se tiene:

\section{Condiciones para el contexto}

El contexto para la situación problema, se plantea desde lo cotidiano mediante un texto que involucra unas tareas que traen consigo unas preguntas no matemáticas, que al querer ser abordadas desencadena nuevas preguntas que involucran al estudiante en el uso de una trama de conceptos, representaciones y procedimientos matemáticos, que están en relación con un conocimiento básico que se pretende, consciente e 
intencionalmente, poner en juego, en relación con los ejes conceptuales que proponen los lineamientos curriculares para el área de matemáticas y que están en correspondencia con los que cita el ICFES. El contexto así planteado, surge como el pretexto para que los estudiantes asuman la actitud de enfrentar las preguntas o tareas planteadas mediante el uso de conocimiento matemático, de tal manera que se posibilite la construcción de un nuevo conocimiento matemático escolar.

\section{Formas de la actividad y de la acción en el aula}

A partir de considerar las formas de la actividad y de la acción en el aula como un conjunto de normas y reglas establecidas para regular y orientar las formas de trabajo durante el proceso ocurrido al abordar la situación problema, es necesario pensar que desde ellas es posible direccionar el proceso metodológico que se desea llevar a cabo durante el desarrollo de la situación problema. En relación con lo anterior, hay que plantear condiciones que estén referidas a determinar en primer lugar las responsabilidades de los estudiantes, profesores y el papel del conocimiento, y en segundo lugar, las formas de proceder o interactuar entre ellas. Estas formas de la actividad y de la acción en el aula, ofrecen normas metodológicas que deben estar presentes en el accionar durante la búsqueda de explicación de la situación problema.

\section{Responsabilidad del profesor}

El profesor como orientador de la situación problema, fundamenta su quehacer en el aula en:

- Concebir y presentar la situación problema.

- La formulación de preguntas a los estudiantes, tendientes a que ellos amplíen, aclaren o verifiquen lo que dicen o lo que les dicen, de tal manera que el ambiente de clase propicie la valoración del aprendizaje y uso del conocimiento matemático.

- Propiciar el trabajo cooperativo y de institucionalización entorno a las acciones ejecutadas para dar explicación a las preguntas propuestas.

- Tener en cuenta la pertinencia de las participaciones de los estudiantes desde las pretensiones de la actividad, de tal manera que desde su ser maestro regule el desarrollo de la situación para que no sea desviada.

- La mediación en las discusiones y confrontaciones que se den entre los alumnos en torno al problema tratado y sus posibles soluciones.

- Su papel de animador y moderador de la situación problema, interviene en tanto sea necesario para enriquecerla y dinamizarla.

- Promover y proponer preguntas que propicien que los estudiantes validen lo que hacen, en aras de fortalecer aciertos y descartar los desaciertos, de tal forma que desde ellos se reoriente el trabajo; por tanto, es uno de los actores fundamentales en el proceso de institucionalización del saber.

\section{Responsabilidad del estudiante}

El estudiante, como protagonista en la situación problema, debe centrar su accionar dentro del aula en las siguientes acciones:

- Gestar y ser responsable de las alternativas de solución que se proponen y producen en relación con las preguntas y/o tareas formuladas en el texto que se presenta. 
- Validar los resultados aparecidos en el proceso de solución, es decir quién acepta o rehúsa las proposiciones de los otros compañeros o del profesor.

- Aportar desde las matemáticas, conceptos, procedimientos y representaciones que le permitan abordar la situación problema.

- Apropiarse de la situación problema, es decir, hacerla suya, esto luego de la presentación que hace el profesor, de tal manera que no aparezca como una tarea por cumplir, sino una responsabilidad para asumir.

- Evaluar, cuestionar y criticar las sugerencias o ideas que presenten el profesor como los mismos estudiantes.

- Construir nuevo conocimiento a través de procesos de uso de conocimientos ya presentes en el estudiante.

- Ser el responsable de promover el desarrollo de una comunidad cooperativa en el aula, donde él participe activamente en el desarrollo y monitoreo de la situación problema.

\section{Papel del conocimiento matemático escolar}

El conocimiento, como objeto de aprendizaje y desde las pretensiones de la propuesta, sustenta su papel en las siguientes consideraciones:

El conocimiento matemático escolar es considerado como un instrumento o referente que al ser usado brinda alternativas de explicación a la situación problema que se presenta en el aula, es un referente para validar tanto los procesos, representaciones y conceptos matemáticos usados en el proceso de búsqueda de la explicación de la situación problema.
En relación con las forma de la acción en el aula de clases, se necesita al momento de abordar la situación problema explicitar unas reglas que direccionen las relaciones e interacciones entre estudiante-conocimiento-profesor, de tal manera que se posibilite una nueva cultura de trabajo en el aula mediante la cual se propicie la creatividad, el trabajo solidario, la autonomía, la investigación, la innovación y la mejor formación de los estudiantes; y en la cual el estudiante mantenga un estado actitudinal de uso del conocimiento.

\section{Proceso de búsqueda de explicación}

La situación problema, al ser abordada, propicia en el estudiante una actitud de búsqueda de explicación para la comprensión del contexto, sus interrogantes o afirmaciones que al ser asumidas en diferentes momentos hacen que la situación se torne dinámica.

Un primer momento ocurre cuando el estudiante desde su afectividad y desde lo cotidiano, asume la situación como suya de manera natural y con la cual se siente comprometida al buscarle explicación mediante acciones interpretativas. En este sentido la situación problema nace para el estudiante cuando reconoce lo que necesita hacer, dónde ir y cómo ir, en relación con el conocimiento matemático disponible. Un segundo momento, ocurre cuando el estudiante al buscar explicación y respuestas a los interrogantes del contexto, propicia el crecimiento de la situación mediante la aparición de acciones argumentativas que posibilitan el intercambio de interpretaciones desde las matemáticas, de tal manera que se inicia un proceso continuo de justificación, donde el conocimiento matemático se convierte en un instrumento facilitador para explorar de mejor manera la realidad presentada en el contexto al transformar lo cotidiano en un proceso académico. 
El último y tercer momento, en este proceso de búsqueda de explicación, transcurre cuando se reproducen nuevas situaciones que resultan de los procesos y acciones hasta el momento llevadas a cabo por los estudiantes. Es aquí, donde la situación problema se desdobla en nuevos problemas que se convierten en objeto de reflexión como unidades de trabajo independiente y que, al ser integrados se constituyen como un todo en relación con la situación problema, de tal manera que desde ellos la situación se transforma, posibilitando que los estudiantes trascienden las pretensiones de la actividad.

\section{Espacio de evaluación}

Es el espacio en el cual se analizan y valoran las acciones de tipo interpretativo, argumentativo y propositivo, ejecutadas por el estudiante en su intento de buscar explicaciones para la situación problema; esta evaluación, se realiza continuamente en el transcurrir de la situación mediante distintas formas y en varios momentos, está dirigida a la recolección y aporte de información que permitan evidenciar el saber-hacer en matemáticas en lo relacionado con la comprensión, práctica y uso de conceptos, terminología, notación, destrezas, razonamiento y estrategias matemáticas.

\section{Institucionalización del saber}

Este se entiende como el momento en el cual el docente o los estudiantes desde el eje conceptual hacen precisiones y puntualizaciones, respecto a los conceptos, representaciones y procedimientos surgidos a partir del trabajo hecho por los estudiantes en el proceso de búsqueda de explicación a la situación problema. Además es aquí donde el profesor como orientador de la actividad, organiza, aclara, amplía, profundiza y sintetiza las ideas o aportes hechos por los estudiantes.

\section{Metodología}

Esta experiencia tuvo lugar en el grado 6o de la Institución Educativa La Unión de Sincelejo, se contó con un grupo de 35 estudiantes, quienes participaron de la experiencia en el marco de la investigación - acción.

La información del acontecer de la experiencia se registró, mediante técnicas como la observación directa, diarios de campo, igualmente las entrevistas que se realizaron en el transcurso del desarrollo de la experiencia.

\section{La metodología para el trabajo de aula: los momentos y la estructura de la actividad}

Para iniciar el trabajo en el aula, se presentó una charla sobre las relaciones personales, la convivencia familiar y sus múltiples efectos, se contó con la colaboración de la oficina de bienestar estudiantil de la institución, luego en una clase posterior se presentó el siguiente texto a los estudiantes:

En los últimos años y en actualidad, existe la tendencia en la Institución Educativa La Unión, que el rendimiento académico y el interés por el estudio de los grados 6o es insuficiente, se nos ocurre entonces hacernos la pregunta: ¿Será que las relaciones familiares y la convivencia familiar de los estudiantes influyen en esta situación?

El texto anterior, fue el pretexto para que los estudiantes generaran la situación problema e iniciar el proceso metodológico de la actividad, el cual se espera desarrollar en varios momentos que se generaron en el transcurso de la actividad.

Un primer momento en el desarrollo de la situación problema, fue el abordaje de la situación de manera natural, en el cual el profesor mediante cierto tipo 
de preguntas orientó la discusión de los estudiantes para que reconozcan de manera consciente y responsable la necesidad de abandonar la actitud "superficial" frente a la situación que se plantea y adquieran el compromiso de apropiarse y comprender la temática relacionada con los conceptos como el de rendimiento escolar, convivencia, integración familiar, o interés estudiantil, variables que intervienen en el problema.

En este momento se motivó a los estudiantes para que asumieran el compromiso de hacer ese estudio, que permitiera determinar la necesidad ó no de hacer el estudio, lo cual se promovió con estas preguntas: ¿Qué tanto influye la convivencia familiar con mi rendimiento académico? ¿Estamos realmente interesados por estudiar? ¿Qué necesitamos a nivel familiar para rendir académicamente?

Una vez logrado lo anterior, se pasó a un segundo momento, denominado momento de búsqueda en el cual se formularon preguntas que conllevaron al estudiante a reflexionar sobre lo que había que hacer, a dónde ir, qué acciones ejecutar, de tal manera que se pudiera llegar a comprender la complejidad que involucraba el querer conocer el efecto que tienen las relaciones familiares en su rendimiento académico.

En ese momento se pretendía que el estudiante determinara la metodología que se iba a seguir para darle explicación a los interrogantes que se habían planteado. Estas reflexiones estaban orientadas por las preguntas: ¿A dónde hay que ir a consultar información? ¿Hablar con profesores? ¿Con la trabajadora social? ¿Buscar libros? ¿Qué libros o qué tipo de información?

Agotado ese momento, se siguió con un tercer momento denominado revisión bibliográfica, en el cual los estudiantes llevaron a cabo una indagación teórica donde puntualizaron e interpretaron los conceptos (variables) involucrados al pretender conocer sobre el rendimiento académico y su vinculación con la familia. Así mismo, el estudiante pudo entrar en conversaciones con profesores de otras áreas en aras de complementar y profundizar los conocimientos que puntualizaba.

Terminado este momento apareció un cuarto momento denominado Necesidad de Información en el cual los estudiantes ubicaron y recogieron la información que requían, como también las formas posibles de obtenerla. En relación con esto se propuso los instrumentos más pertinentes que les facilitaran la recolección de la información, para después, iniciar el momento de Sistematización, en el cual reflexionaron en torno a cómo organizar y presentar la información, de tal manera que se facilite la comprensión y el análisis de la información.

Un sexto momento fue la Interpretación de la Información en el cual el estudiante desde las matemáticas comparó la información, tablas, gráficas y en general todos los datos que le permitieron conocer el estado académico de los estudiantes $y$, a la vez, lo posibilitaron para tomar decisiones en torno al estudio que se efectuaba y un momento de cierre, en el cual se sistematizó todo el conocimiento construido y usado, se puntualizó en cada uno de los conocimientos matemáticos aparecidos y reconocieron las acciones y quehaceres que posibilitaron usar los conocimiento en el desarrollo de la situación, a partir de los estudios elaborados y de las conclusiones.

\section{Otras orientaciones que describen la metodología del trabajo de aula}

Si bien, lo descrito anteriormente hace parte de las acciones metodológicas de la actividad en el aula, es necesario agregar 
otros elementos que ayuden a conocer en concreto, cómo se encaminó el trabajo de los estudiantes, al desarrollo de la competencia comunicativa en matemática, en sus manifestaciones interpretativa, argumentativa y propositiva.

Es importante tener claro que la situación problema que se generó en el trabajo tiene como eje específico el de aleatoriedad y se centró en la construcción de conocimientos básicos estadísticos, en este caso; las medidas de tendencia central: media, moda, mediana, para ello se hizo necesario contar con conocimientos previos en relación con los conceptos de muestra, frecuencia, unidad de medida, población; representaciones en tablas, gráficas, diagramas y procedimientos de recolección de datos, tabulación, y sistematización de datos; para ello se asumió la metodología de trabajo de grupo, para recurrir a la revisión de estos conceptos, para dar respuesta a la pregunta problema, por medio el uso y la aplicación de conocimientos matemáticos

La dinámica a seguir en el trabajo de aula se detalla a continuación:

1. La actividad se desarrolla individual y grupalmente y finalizará con la entrega de un informe escrito por cada grupo.

2. Se conforman grupos de trabajo, que deben preparar informes parciales de avances y dificultades, para ser expuestos en una plenaria. Estos grupos deben ser de dos o tres personas como mínimo o máximo.

3. En cada grupo se nombra un relator, quien debe dar cuenta por escrito de todo lo sucedido en el grupo, a saber:

- Discusión sobre las posiciones individuales
- Puntos de acuerdos y desacuerdos

- Discusión sobre los puntos de descuerdo

- Consensos obtenidos por el grupo en cuanto a ideas, conceptos y conocimiento revisado y construido (matemático y no matemático)

- Puntos que quedan pendiente por no llegar a un consenso.

- Preguntas que surgen en medio de la actividad y compromisos.

4. Al iniciar las exposiciones de los grupos, el grupo expositor (grupo E), presenta al resto de curso (grupo D) lo que ha hecho en el proceso de búsqueda de explicación, empleados los recursos que se crea necesario (cartel, video beam o audio entre otros).

5. Debe discutirse y acordarse con todo el curso las condiciones para la intervención de los estudiantes, procurar que los acuerdos sean:

- El grupo E hace su exposición y procura explicitar los términos que intervengan en ella.

- El grupo D sigue la exposición sin intervenir, pero cada uno de los otros grupos debe preparar al menos una pregunta para el grupo $\mathrm{E}$, encaminada a la comprensión de la exposición.

- Las preguntas son escritas en el tablero por el grupo $\mathrm{E}$ y se deben contestar desde sus posibilidades. Cuando sus argumentos no sean aceptados, termina la exposición y se compromete a dar la respuesta en la próxima sesión.

- No se permiten opiniones personales, ni juicios de valor. El profesor debe estar atento a tales situaciones y pedir en tal caso se elabore la pregunta correspondiente. 
- El profesor es un integrante más del grupo $D$ y debe intervenir con las preguntas que crea conveniente para lograr que el grupo E elabore lo necesario para el desempeño adecuado de su labor.

- El profesor debe estar atento a la participación del grupo D, y propiciar la pregunta.

6. Se nombran tres o cuatro evaluadores para la exposición.

7. En este momento puede intervenir otro grupo, y se aplica la misma metodología.

8. Cada grupo, puede continuar su exposición en la medida que esté preparado.

9. El profesor debe estar pendiente de los avances y obstáculos, con el fin de dar orientaciones pertinentes y facilitar la bibliografía adecuada.

10. Cada grupo, prepara una carpeta que contenga las relatorías y el trabajo producido.

11. Todos los grupos, deben hacer una lista con las preguntas que surjan en las distintas exposiciones, y contestarlas en la medida que avance en sus consultas.

12. La exposición de cada grupo debe evolucionar en el tiempo, con respecto a las anteriores.

13. Cuando el conocimiento circulante en el curso y conseguido en el proceso de consulta y revisión bibliográfica, no sean suficientes para desarrollar la actividad, el docente asignará otra actividad o actividades, cuyos resultados servirán para retomar la primera actividad y hacer puntualizaciones con respecto a las temáticas tratadas.

\section{Análisis y resultados}

La información que se recolectó, sistematizó y analizó de acuerdo con los elementos y estructura de la situación problema propuesto y gira en función de los siguientes aspectos: las condiciones para el contexto, las formas de la actividad y la acción en el aula, los procesos de búsqueda de explicación, el espacio de evaluación y la institucionalización del saber.

\section{Los observables}

En el estudio se tomó en cuenta las acciones ocurridas en la interacción entre estudiante, saberes y profesor, en el trabajo de aula, de tal manera que desde ellas y ellos, se pueda dar cuenta de todo aquello que aconteció. Estas acciones se denominan observables, y son, para la investigación, parámetros que direccionarán el proceso de investigación. Los estudiantes y el docente en el hacer de aula expresan sus ideas mediante palabras, escritos, gestos y otras acciones que están enmarcadas en el discurso y en la actitud que tiene cada uno, por tanto, se propone estos dos elementos, discurso $y$ actitud, como los observables para dar cuenta, tanto del tipo de interacción en el aula, como de los saberes construidos y la competencia desplegada. La competencia comunicativa, particularmente en matemática, encuentra en el discurso un espacio amplio para desarrollarse y manifestarse, además a través de él es posible:

- Conocer conceptos, representaciones y procedimientos que desde las matemáticas los estudiantes ponen en juego.

- Analizar las preguntas y afirmaciones hechas por los estudiantes en relación con la complejidad o simplicidad que encierre. 
- Apreciar las formas como los estudiantes validan, argumentan y sustentan las ideas y procederes.

- Evaluar los hechos y acciones de los estudiantes ante la situación planteada.

- Determinar si se están usando los conocimientos para interpretar, argumentar, y proponer en el transcurrir y evolucionar de la situación problema.

- Observar cómo los estudiantes amplían o aclaran lo que escuchan o dicen.

- Conocer y acercarse a los momentos de significación y búsqueda de sentido que los estudiantes vivencian en el proceso de abordar la situación.

La actitud como observable en la interacción de la tríada estudiante-saberprofesor, permite tener indicadores que den cuenta de los comportamientos, actuaciones y procederes que tienen los estudiantes en el trabajo de aula durante el desarrollo de la situación problema. Desde este observable es posible:

- Analizar la actitud e interés del estudiante frente a la situación problema mediante su trabajo, argumentos y propuestas.

- Visualizar las acciones de tipo interpretativo, argumentativo y propositivo y las formas como se manifiestan.

- Apreciar las formas de trabajo que los estudiantes ponen en práctica y el compromiso con cada una de ellas.

- Conocer la forma como estudiante y docente reaccionan ante preguntas 0 ideas expuestas durante el desarrollo de la situación.

- Determinar si los estudiantes asumen comportamientos en relación con el uso y construcción de conocimiento, a partir de su disposición de interpretar, argumentar y proponer.
- Observar los roles que asumen los estudiantes tanto en su trabajo individual como grupal.

- Observar las formas de trabajo de los estudiantes y sus procederes en cada uno de los momentos que tiene la situación problema.

A través de la experiencia, se evidenciaron algunos actos ejecutados y observados en cada uno de los momentos, que reflejaron el desarrollo de la competencia comunicativa en matemáticas: actos interpretativos, argumentativos y propositivos. Dentro de los resultados destacables se tienen, por ejemplo, que en los momentos de abordaje de la situación y búsqueda de explicación la totalidad de estudiantes se sintieron comprometidos con la situación, se mostraron interesados ya que la problemática abordada forma parte de la realidad que viven. Los estudiantes realizaron acciones como: preguntar, consultar, revisar, que evidencian actitudes de interés y esmero por darle solución a la situación. Igualmente se propició el crecimiento de la situación, mediante la aparición de acciones argumentativas y propositivas que posibilitan el intercambio de interpretaciones desde las matemáticas, surgieron nuevas preguntas y cuestionamientos que implicaron acudir a otras disciplinas como sociales, sicología, trabajo social y nuevas tecnologías promovida así la interdisciplinariedad del aprendizaje. Igualmente para los momentos deinstitucionalización y la evaluación del saber, donde a través del informe que cada grupo de estudiantes entregó al final de cada sesión de trabajo, se evidenció, el uso del conocimiento matemático en y particular el estadístico. Se pudo construir conocimiento en relación con la temática, además que se ampliaron los conocimientos previos. En toda la actividad se generó y propició procesos de aprendizaje a través de la acción, los 
estudiantes asumieron la problemática de su rendimiento académico como una actitud natural y de compromiso para su propia superación, además lograron vincular también a la familia en el estudio, alertándola sobre su responsabilidad con su aprendizaje y formación en la institución.

\section{Conclusiones}

- El articular y poner en correspondencia las acciones de aula con la nueva evaluación por competencias propuestas por el ICFES exige que desde el aula se ejecuten actividades que posibiliten en el estudiante el uso de sus conocimientos, para desde ellos darle sentido al mundo que lo rodea.

- Las situaciones problemas como una estrategia de trabajo en el aula lleva implícita la necesidad de reorganizar y reestructurar los currículos existentes de tal forma que desde ellos se propicie en los estudiantes una actitud natural y continua de interpretar, argumentar y proponer.

- El pretender concebir una situación problema que sea rica y atractiva para los estudiantes, pone de manifiesto la necesidad de hacer aparecer múltiples ideas que no sólo estén en las matemáticas, por tanto, su carácter de actividad interdisciplinar.

- Al asumir la situación problema como dinámica de trabajo para el aula el papel del profesor es determinante para conseguir lo que se pretende, lo que le exige mayor preparación y compromiso social con su labor.

- El trabajo de aula alrededor de las situaciones problema se constituye en una prometedora opción para los maestros colombianos no sólo para evaluar competencias sino para desarrollarlas.

- La situación problema como proceso dinámico de construcción de conocimiento tiene su esencia en el trabajo de aula donde surgen múltiples situaciones que son imprevistas y que de alguna manera dificultan que los estudiantes alcancen los logros que se querían, pero le posibilitan acceder a conocimientos que no se esperaban.

- El anterior estudio no es el final de ningún proceso,, se espera que se constituya en el punto de partida para continuar un estudio más profundo, alrededor del marco del desarrollo de competencias a través de situaciones problemas y del trabajo de aula.

\section{Referencias}

Beltrán, S. (1998). Un acercamiento histórico a la resolución de problemas. Bogotá, Colombia.

Bonilla, M. (1999). La enseñanza de la aritmética escolar y la formación del profesor: cuadernos de matemática educativa. Bogotá, Colombia: Gaia.

Calderón, D. (2001). Requerimientos didácticos y competencias argumentativas en matemáticas. Bogotá, Colombia: Colciencias.

Colombia, MEN. (1998). Serie Lineamientos Curriculares. Matemáticas. Bogotá, Colombia: Instituto Colombiano para el Fomento de la Educación Superior.

Colombia, MEN (1997). Evaluación de logros en matemáticas. Serie publicaciones para maestros - SNE-. Bogotá, Colombia: El Ministerio.

Hernández, C. (1998). Exámenes de Estado: Una propuesta de evaluación por competencias. Serie Investigación y Evaluación Educativa -SNP Bogotá, Colombia:. ICFES.

ICFES. (1999). Nuevo Examen de Estado. Matemáticas. Serie publicaciones para maestros.-SNE- Bogotá, Colombia: El Ministerio.

Santos, L. (1997). Principios y métodos de la resolución de problemas en el aprendizaje de las matemáticas. México: Iberoamericana. 